\title{
Mathematical modeling for the evaluation of various parameters of 5-methyl salicylaldehyde aniline nano composite using fuzzy evidence theory
}

\author{
R. Irene Hepzibah ${ }^{1,4}$, G. Geethalakshmi ${ }^{2}$, R. Ida Malarselvi ${ }^{3,4}$ \\ ${ }^{1}$ Department of Mathematics, AVC College of Engineering, Mayiladuthurai- 609305, Tamil Nadu, India \\ 2 Department of Mathematics, K.S.K College of Engineering and Technology, Kumbakonam, \\ Tamil Nadu, India \\ ${ }^{3}$ Centre for Advanced Material Research, Government Arts College, Kumbakonam, Tamil Nadu, India \\ ${ }^{4}$ Research and Development Centre, Bharathiar University, Coimbatore - 641046, Tamil Nadu, India \\ ireneraj74@gmail.com, geethavish294@gmail.com, idamalaiselvi@gmail.com
}

PACS 02.10Ab, 02.50Cw, 02.50.Le, 07.05.Mh

DOI 10.17586/2220-8054-2016-7-3-518-522

\begin{abstract}
Densities, viscosities, surface tension and Ultrasonic speeds of nano composite 5- Methyl Salicylaldehyde (5MS) with Aniline (A) were measured over the entire composition range at temperature $303 \mathrm{~K}$. The adiabatic compressibility $(\beta)$, free length $\left(L_{f}\right)$, free volume $\left(V_{f}\right)$ and viscous relaxation time $(T)$ have been calculated from the experimental data. The experimental results have been correlated using Fuzzy Evidence Theory and the results are interpreted on the basis of possible hydrogen bonding between unlike molecules and changes in molecular association equilibria as well as structural effects for these systems. A good agreement among experimental data and the values estimated by theoretical procedure was obtained.
\end{abstract}

Keywords: 5-MS-A composite material, hydrogen bonding, acoustical and physical parameters, Fuzzy Evidence Theory.

\section{Introduction}

Organic liquid state is a fascinating area of current research. The term composites are now well-established in the understanding of such organic liquids. In many industrial applications, liquid mixtures are used in processing and product formulations. Studies on viscosity and acoustic properties of liquid composites consisting of 5 MS with aniline are of interest because of their use as dye carrier formulations in textile processing and pharmaceutical industry. These properties are of considerable interest in understanding the intermolecular interactions in liquid mixtures. Yet, a unified understanding of the mixing behavior of liquids is still controversial. In this respect, it is of interest to further carry out the systematic investigations of these properties for a better knowledge of the molecular interactions. A very large number and variety of intermolecular interactions have been used in composites. Most notable among such interactions are hydrogen bonding which arises from the non-polar regions of aromatic molecules and very effective design element in composite materials. Much research has compared the experimental values of interaction parameters with theoretically calculated values for composites using different models. G. M. Badger and A. G. Moritz [1] examined the $\mathrm{C}-\mathrm{H}$ stretching of methyl groups attached to polycyclic aromatic hydrocarbons. V. C. Farmer and R. H. Thomson [2] studied the inter- and intramolecular hydrogen bonding in anilines. Infrared studies on the self-association of chloroform were given by Edwin D. Becker [3] in 1950. J. C. Evans [4] proposed the vibrational assignments and configuration of aniline, aniline NHD and ND2. In spite of technological importance of 5MS-A no such studies on spectral and acoustical properties for 5MS-A systems are available in literature. We report here the above said studies of $5 \mathrm{MS}-\mathrm{A}$ with $\mathrm{CCl}_{4}$ at $303 \mathrm{~K}$ over the entire composition range. In the present work, an attempt has been made to employ fuzzy evidence theory for optimizing the key influencing parameters in the formation of 5MS-A composite.

\section{Experimental}

\subsection{Materials and measurements}

Analytical grade reagents were used without any further purification. The infrared spectrum of the title mixture was recorded using PERKIN ELMER spectrum RX FT-IR system. The 1H-NMR spectrum of title mixture was performed in $\mathrm{CDCl} 3$ by using BRUKER AC 400 (400 MHz) Spectrometer. The sound speed was measured using a Single crystal Ultrasonic Interferometer at an operating frequency of $2 \mathrm{MHz}$. The viscosity was measured using an Oswald's Viscometer calibrated with double distilled water. The density was measured by a $10 \mathrm{ml}$ specific gravity bottle calibrated with double distilled water and acetone. Refractive indices were measured with an Abbe refractometer (Erma A 302A) and the values were obtained for Na-D light with an error less than \pm 0.0002 units. Surface Tension was measured using the pendent drop method. 


\subsection{Synthesis of $5 \mathrm{MS}$ with aniline in $\mathrm{CCl} 4$ solvent}

Materials having moderate to high solubility at temperature ranging from room temperature to $303 \mathrm{~K}$ at atmospheric pressure form complexes when their samples were carefully mixed. In the present systems, the various concentrations of the ternary liquid mixtures were prepared in terms of mole fractions, out of which, the mole fractions for the first component 5-MS varied from 0.01 to $0.05 \mathrm{ml}$ and the second component aniline were varied from $\mathrm{ml}$. The required solute concentrations of the above-title mixture were prepared in $5 \mathrm{ml} \mathrm{CCl}_{4}$. The formation of the complex was noticed by its pale color and also the absence of solid precipitates.

\section{Results and discussion}

\subsection{Spectroscopic characterization}

FT-IR spectra of newly synthesized composite material were recorded from $\left(400-4000 \mathrm{~cm}^{-1}\right)$. All the above spectral data confirm the formation of the composite due to intermolecular hydrogen bonding. The relevant work was done by Ramachandra Raja. C et.al. [5] in 2013 and Maneesh Sharma et.al. [6] in 2012. The 300 MHz proton NMR spectrum of the synthesized composite 5MS-A was measured in $\mathrm{CDCL}_{3}$ using Bruker instrument. The signals observed in the NMR spectra were in good agreement with reported values in the literature. The relevant work was done by Ida Malarselvi R. et.al. [7] in 2014.

\subsection{Molecular acoustical and physico-chemical behavioural studies}

In the composite, the ultrasonic velocity increases with the increasing concentration of 5MS-A. The variation of sound speed in a solution depends upon the increase or decrease of intermolecular free length (Lf) after mixing the compounds. A reduction in adiabatic compressibility is an indication that component molecules are held closely to each other. The internal pressure of the new material in the present study, which increases with increased concentration of 5-MS, can be attributed to there being definite interaction present between the different components of the mixture. The acoustic impedance $(Z)$ of a material is the opposition exerted by the medium particles to sound energy. In the present system, the cohesive energy (CE) increases and the relaxation time decreases, which shows that the intermolecular energy is so high in the new composite material. The viscosity increases as the molar concentration of the solute mixture increases up to $100 \mathrm{~mole} \%$ and further increase of the mole fraction of the solute mixture it still increases, which shows the strong solute-solvent interactions in the title mixture. The density is ever increasing and the refractive index decreases due to the formation of strong hydrogen bonding and the molecular association of the new material. Xinghua Su et.al. [8] in 2012 pointed out the same relative work. Due to the strong intermolecular interactions, the surface tension decreases with the increased mole fraction value. The intermolecular forces are attractive in nature. The molecules of the 5MS-A composite are strongly attracted by the liquid molecules. Concentration at the surface layer of the liquid results, in a decrease in the surface electrons and brings about a decrease in surface tension. Similar results were reported by K. Prasad et.al. [9] in 2006 and M. V. Rathnam et.al. [10] in 2012. See also [11-15].

TABLE 1. Mole Fractions of 5MS $\left(X_{1}\right)$, Mole Fractions of Aniline $\left(X_{2}\right)$, Values of Velocity $(C)$, Density $(P)$, Viscosity $(N)$, Refractive Index $(D)$,Surface Tension $(T)$ and BPA

\begin{tabular}{|c|c|c|c|c|c|c|}
\hline$X_{1}$ & $X_{2}$ & $C \mathrm{~ms}^{-1}$ & $i \rho \mathrm{kgm}^{-3}$ & $\eta \times 10^{3} \mathrm{Nsm}^{2}$ & $D$ & $T \times N / m$ \\
\hline 0.04 & 0.0273 & 641 & 1.5852 & 0.00116 & 5.9877 & 0.0134668 \\
\hline 0.08 & 0.0547 & 724 & 1.5920 & 0.00127 & 2.9021 & 0.0162094 \\
\hline 0.12 & 0.0820 & 772 & 1.6183 & 0.00151 & 1.7048 & 0.0151904 \\
\hline 0.16 & 0.1094 & 906 & 1.6239 & 0.00173 & 1.2424 & 0.0162238 \\
\hline 0.20 & 0.1368 & 1047 & 1.6415 & 0.00186 & 0.9045 & 0.0162652 \\
\hline 0.24 & 0.1436 & 1065 & 1.6521 & 0.00192 & 0.9028 & 0.0162785 \\
\hline 0.28 & 0.1572 & 1079 & 1.6635 & 0.00205 & 0.9006 & 0.0162964 \\
\hline 0.32 & 0.1625 & 1104 & 1.6743 & 0.00214 & 0.8924 & 0.0170562 \\
\hline
\end{tabular}




\section{Dempster-Shafer theory (DST) of evidence}

Dempster-Shafer Theory (DST) is a mathematical theory of evidence. In a finite discrete space, DempsterShafer theory can be interpreted as a generalization of probability theory where probabilities are assigned to sets as opposed to mutually exclusive singletons. In traditional probability theory, evidence is associated with only one possible event. In Dempster-Shafer Theory, evidence can be associated with multiple possible events.

A frame of discernment, (or simply a frame), is usually denoted as a set of mutually exclusive and exhaustive propositional hypotheses, of which, one and only one is true [8]. Evidence theory is based on two dual non-additive measures, namely Belief measure and Plausibility measure. There is one important function is Dempster-Shafer theory to define Belief measure and plausible measure which is known as Basic Probability Assignments.

TABLE 2. Mole Fraction of 5MS $\left(X_{1}\right)$, Mole Fraction of Aniline $\left(X_{2}\right)$, Values of Adiabatic Compressibility $(\beta)$, Internal Pressure $\left(\pi_{1}\right)$, Cohesive Energy $(C E)$, Acoustic Impedance $(Z)$, Free Length $\left(L_{f}\right)$ and Relaxation Time $(T)$

\begin{tabular}{|c|c|c|c|c|c|c|c|}
\hline$X_{1}$ & $X_{2}$ & $\begin{array}{c}i \beta \times 10^{-10} \\
\mathrm{M}^{2} \mathrm{n}^{-1}\end{array}$ & $\begin{array}{c}\pi_{i} \times 10^{6} \\
\mathrm{~nm}^{-2}\end{array}$ & $\begin{array}{c}C E \\
\mathrm{~kJ}^{\mathrm{mol}}\end{array}$ & $\begin{array}{c}Z \times 10^{6} \\
\mathrm{Kgm}^{-2} \mathrm{~s}^{-1}\end{array}$ & $\begin{array}{c}L_{f} \times 10^{-10} \\
\mathrm{~m}\end{array}$ & $\begin{array}{c}\tau \times 10^{-12} \\
\mathrm{~s}\end{array}$ \\
\hline 0.04 & 0.0273 & 7.0092 & 3.8124 & 19.1440 & 1.0161 & 46.3507 & 0.2020 \\
\hline 0.08 & 0.0547 & 6.9793 & 3.6564 & 36.7160 & 1.1526 & 47.0367 & 0.1851 \\
\hline 0.12 & 0.0820 & 6.8691 & 3.6375 & 53.8885 & 1.2493 & 47.4595 & 0.1587 \\
\hline 0.16 & 0.1094 & 6.8422 & 3.4015 & 66.9709 & 1.4712 & 48.0739 & 0.1388 \\
\hline 0.20 & 0.1368 & 6.7688 & 3.2329 & 78.7204 & 1.7186 & 48.5914 & 0.1307 \\
\hline 0.24 & 0.1436 & 6.6790 & 3.1100 & 89.3542 & 1.9321 & 49.0433 & 0.1295 \\
\hline 0.28 & 0.1572 & 6.5430 & 3.0125 & 98.0032 & 2.1096 & 49.6024 & 0.1254 \\
\hline 0.32 & 0.1625 & 6.4852 & 2.8955 & 107.9962 & 2.3061 & 50.1278 & 0.1195 \\
\hline
\end{tabular}

A function $m: 2^{\Theta} \rightarrow[0,1]$ is called Basic Probability Assignments (BPA) on the set $\Theta$ if it satisfies the following conditions:

$$
\begin{gathered}
m(\phi)=0, \\
\sum_{A \subseteq \Theta} m(A)=1,
\end{gathered}
$$

where $\phi$ is an empty set and $A$ is any subset there of.

The Basic Probability Assignment function (or mass function) is a primitive function. Given a frame, $\Theta$, for each source of evidence, a mass function assigns a mass to every subset of $\Theta$, which represents the degree of belief that one of the hypotheses in the subset is true, given the source of evidence. A subset A of a frame $\Theta$ is called the focal elements of $m$, if $m(A)>0$.

The lower bound, Belief for a set $A$ is defined as the sum of all the basic probability assignments of the proper subsets $(B)$ of the set of interest $c(A)(B \subseteq A)$. The upper bound, Plausibility is the sum of all the basic probability assignments of set (B) that intersect the set of interest $(A)(B \cap A \neq \phi)$. Formally for all sets $A$ that are elements of the power set $A \in P(X)),[16], \operatorname{Bel}(A)=\sum_{B / B \subseteq A} m(B)$ and $P l(A)=\sum_{B / B \cap A \neq \phi} m(B)$.

The two measures, Belief and Plausibility are non-additive. This can be interpreted to mean that it is not required for the sum of all the Belief measures to be one, and similarly for the sum of all the Plausibility measures. Hence, interval $[\operatorname{Bel}(A), P l(A)]$ is the range of belief $A$.

\subsection{The dempster rule of combination}

The Dempster rule of combination is critical to the original conception of the Dempster-Shafer theory. The measure of Belief and Plausibility are derived from the combined basic assignments. Dempster's rule combines multiple belief functions through their basic probability assignments (m). These belief functions are defined on the same frame of discernment, but are based on independent assignments or bodies of evidence. The Dempster rule of combination is purely a conjunctive operation (AND). The combination rule results in a belief function based on conjunctive pooled evidence [17]. 
Let $m_{1}$ and $m_{2}$ be two mass functions defined on the same frame of discernment, and then a combined BPA can be obtained by using Dempster's combination rule, the combined BPA $m=m_{1} \oplus m_{2}$ is defined as follows

$$
m= \begin{cases}\frac{\sum_{B \cap C=A} m_{1}(B) m_{2}(C)}{1-\sum_{B \cap C=\phi} m_{1}(B) m_{2}(C)}, & \forall \phi \subseteq \Theta ; \\ 0, & \text { otherwise. }\end{cases}
$$

\section{Application of evidence theory to the experimental data}

Evidence theory can handle both aleatory and epistemic uncertainty. Three important functions in evidence theory, the basic probability assignment function (bpa), Belief function (Bel) and Plausibility function (Pl) are used to quantify the given variable. As already stated in this paper interval focal elements and their Basic Probability Assignments of two variables are combined by Modified Interval Arithmetical operations. To determine the quantitative formation characteristics of the composite, fuzzy evidence theory was employed to identify the level of importance in interaction parameters on their performance characteristics. The effect of these parameters on interaction has been investigated using Basic Probability Assignments of two variables $X_{1}$ and $X_{2}$ and the variation of this interaction parameter with the mole fraction of composite has been discussed in terms of molecular interaction. The validity of the observed values developed and the results were discussed below in Table 3 . In this study, the sum of Basic Probability Assignments (BPA) is equal to one. The effectiveness of the model has been checked by the validation with experimental values and the molecular interactions and the complex formation evolved through Basic Probability Assignments can successfully predict the complex formation for any combination of the experimental results. The experimental results have been validated by asserting that the predicted values are very close to each other and hence the developed models are suitable.

TABLE 3. BPA

\begin{tabular}{|c|c|c|}
\hline$X_{1}$ & $X_{2}$ & BPA \\
\hline 0.04 & 0.0273 & 0.10 \\
\hline 0.08 & 0.0547 & 0.10 \\
\hline 0.12 & 0.0820 & 0.25 \\
\hline 0.16 & 0.1094 & 0.20 \\
\hline 0.20 & 0.1368 & 0.15 \\
\hline 0.24 & 0.1436 & 0.05 \\
\hline 0.28 & 0.1572 & 0.10 \\
\hline 0.32 & 0.1625 & 0.05 \\
\hline
\end{tabular}

\section{Conclusion}

The newly synthesized potential 5MS-A composite with optimum physical and acoustical properties are expected to find a special position in the biomedical field like antibiotic and Anti-elementic agent. 5MS based on 5MS-A were expected to possess a higher co-efficient of viscosity, density and hence may be used in the synthesis of dyes. Dempster's rule combines multiple belief functions through their basic probability assignments and the model can be successfully related with the above process parameters by the molecular interactions and the complex formation.

\section{Acknowledgements}

This work was financially supported by University Grants Commission, Govt of India, New Delhi, within the Minor Research Project scheme under the approval cum sanction No.F MRP-5150/14 (SERO/UGC). All the spectral data were obtained from SAIF, Indian Institute of Technology, Chennai. 


\section{References}

[1] G.M. Badger and A.G. Moritz. Spectrachimica Acta. Pergamon Press Ltd, 1959, 9, P. 672-678.

[2] J.C. Evans. Spectrachimica Acta, Pergamon Press Ltd, 1960, 16, P. 428-443.

[3] Edwin D. Becker. Spectrachimica Acta, Pergamon Press Ltd, 1950, 9, P. 743-746.

[4] V.C. Farmer and R.H. Thomson. Spectrachimica Acta, Pergamon Press Ltd, 1960, 16, P. 559-562.

[5] Maneesh Sharma, Anant A Naik, P.Raghunathan and S.V.Eswaran. J.Chem.Sci., 2012, 124(2), P. $395-401$.

[6] C. Ramachandra Raja and R.Ida Malarselvi. International J. Science and Research (IJSR), 2(1), P. $2319-7064$.

[7] Xinghua Su and Chengwen Qiang, Bull. Mater.Sci., 2012, 35(2), P. 183-189.

[8] R.Ida Malarselvi, C.Ramachandra Raja and Priscilla. R. International J. Science and Research (IJSR), 4(1), P. $2319-7064$.

[9] K. Prasad, K. Siva Kumar, G. Prabhakar, P. Venkateswarlu. Journal of Molecular Liquids, 2006, 123, P. 51-58.

[10] M.V. Rathnam, Reema T.Sayed, Kavita R. Bhanushali, M.S.S.Kumar. Journal of Molecular Liquids, 2012, 166, P. 9-16.

[11] Philbin P, Gordon S. Recent research on the machining of wood- based composite materials. International Journal of Machining and Machinability of Materials1, 2006, 2, P. 186-201.

[12] Gokay Nemli, IsmailbAydin, Emir Zekovic. Evaluation of some of the properties of particle board as function of manufacturing parameters. Journal of Materials and Design, 2007, 28, P. 1169-1176.

[13] Cochran WG, Cox GM. Experimental designs. Wiley, New York, 1992.

[14] Montogomery C., Design and Analysis of experiments, 4-th edition, Wiley Newyork, 1997.

[15] Zhiming Zhang. Interval Valued Intuitionistic Hesitant Fuzzy Aggregation operators and their applications in Group Decision Making. Journal of Applied Mathematics, 2013, 3, Article ID 670285, 33 pages, http://dx.doi.org/10.1155/2013/670285.

[16] George J. Klir, Z. Wang. Generalized Measure Theory. Springer, 1998.

[17] G. Shafer. A Mathematical Theory of Evidence. N. J Princeton University press, 1976. 